\title{
Horehound Oil
}

National Cancer Institute

\section{Source}

National Cancer Institute. Horehound Oil. NCI Thesaurus. Code C107316.

The essential oil of Marrubium vulgare. Horehound oil is used typically in expectorants and cough suppressants. 